\title{
Impactos na produção de políticas "desacopladas" na agricultura norte-americana: o papel do risco
}

Alexandre Bragança Coelho ${ }^{1}$ Erly Cardoso Teixeira ${ }^{2}$

Resumo: Nos últimos anos, esforços têm sido feitos no sentido de modificar as políticas de apoio à agricultura para minimizar os efeitos distorcivos sobre a produção e o comércio internacional. Ênfase tem sido dada às políticas desacopladas de preços e/ou produção, sobretudo às transferências diretas de renda aos agricultores, consideradas neutras em relação às decisões de produção corrente. O argumento é de que estas políticas têm efeitos mínimos na produção atual, pois não influenciam os retornos marginais da cultura, já que seus pagamentos independem da produção corrente.

Este estudo mostra que, ao contrário, as decisões de produção correntes são afetadas pelas políticas de transferência de renda, especialmente através dos efeitos diretos sobre a percepção de risco dos agricultores. Estes efeitos, divididos em efeito-renda e efeito-segurança, estimulam o aumento da área plantada e assim, têm efeitos sobre a produção e os preços.

Palavras-chave: políticas desacopladas, risco, transferências diretas, pagamentos anticíclicos.

Classificação JEL: Q18 e Q12

${ }^{1}$ Doutorando em Economia Aplicada - DER-UFV (Bolsista da CAPES). E-mail: alexandrebc@yahoo.com

2 Professor Titular do Departamento de Economia Rural da Universidade Federal de Viçosa (DER-UFV). E-mail: teixeira@ufv.br 
Abstract: In the last few years, efforts have been made to modify agricultural support policies in order to minimize the distortive impacts on product level and on international trade. Emphasis has been given to decoupled payments, considered neutral to current production decisions. The main argument is that such payments do not influence the crop marginal returns because they are not based on current production.

This article shows, however, that current production decisions are influenced by decoupled payments through direct impacts on risk perception by farmers. These effects, classified as income effect and insurance effect, stimulate the increase in planted area and, therefore, have impacts on production and price levels.

Key-words: decoupled payments, risk, counter-cyclical payments

JEL Classification: $Q 18$ \& Q12

\section{1- Introdução}

As políticas de apoio à produção agrícola têm sido alvos de ataques nos últimos anos pela sua capacidade em distorcer os preços nos mercados internacionais, subvalorizando o investimento agrícola principalmente nos países em desenvolvimento. Pelo acordo da rodada Uruguai do General Agreement on Tariffs and Trade (GATT) em 1994, os países signatários concordaram em diminuir as medidas de apoio à agricultura. No caso dos países desenvolvidos, houve o compromisso de, num período de seis anos, reduzir o valor agregado das medidas de apoio em $20 \%$. Em relação aos países em desenvolvimento, este compromisso de redução foi estipulado em $14 \%$ em 10 anos (TEIXEIRA, 2000).

Entretanto, algumas políticas de apoio foram isentas de redução, constituindo o que se denominou "caixa-verde" (green box). O que caracteriza essas políticas são os impactos mínimos na produção, preços e comércio internacional. Além disso, elas devem atender aos seguintes critérios gerais (BURFISHER \& HOPKINS, 2003):

- O auxílio deve ser financiado pelo governo ao invés dos consumidores; 
- O auxílio não deve ter o efeito de fornecer um suporte de preços aos produtores.

Há critérios específicos que devem ser atendidos para a implementação de cada tipo de apoio. ${ }^{3}$ De forma geral, os exemplos de políticas isentas de redução são as seguintes:

- Pagamentos diretos a agricultores que não dependem da produção corrente ou dos preços atuais;

- Assistência no caso de desastres naturais;

- Políticas de apoio à pesquisa e extensão rural;

- Políticas de proteção ambiental;

- Políticas de ajuda alimentar;

- Políticas de ajuda regional ou a grupos atrasados tecnologicamente;

O interesse deste trabalho está no primeiro item, ou seja, as políticas “desacopladas" (decoupled) da produção e/ou preços correntes. Forçados a diminuir as políticas distorcivas de suporte de preços pelo compromisso da Rodada Uruguai, os países desenvolvidos têm aumentado o montante destinado aos agricultores via políticas 'desacopladas”, especialmente os pagamentos diretos. Do total de auxílio aos agricultores nos países da OECD, o montante transferido via suporte de preço caiu de $78 \%$ em 1986-88 para 68\% em 1999 (OECD, 2001). Grande parte desta queda diz respeito ao crescimento do papel das políticas desacopladas no conjunto de medidas direcionadas à agricultura.

$\mathrm{O}$ argumento é de que estas políticas têm efeitos mínimos na produção atual, pois não influenciam os retornos marginais da cultura, já que seus pagamentos independem da produção corrente. Assim, a decisão de produzir mais, na margem, depende apenas da expectativa dos preços correntes, ou seja, são os sinais de mercado que influenciam a produção e não o auxílio governamental.

Entretanto, esta análise desconsidera os efeitos das políticas desacopladas

\footnotetext{
${ }^{3}$ No caso de transferências diretas de renda, por exemplo, os seguintes critérios devem ser observados: a) a seleção dos agricultores deve observar um critério definido para um período base fixado; b) o montante a ser transferido deve ser independente do volume de produção, preços ou uso de insumo após o período base; c) não deve ser exigido nenhum patamar de produção para que o pagamento seja recebido.
} 
sobre o risco enfrentado pelos produtores, e o conseqüente efeito sobre a produção. Se os produtores são avessos ao risco, uma redução na variância da receita certamente influenciará tanto no total produzido quanto no mix de produtos escolhidos. Além disso, mesmo no caso de pagamentos diretos que não influenciam na variância da receita, o efeito-renda ligado às transferências pode determinar alterações importantes no total produzido. Poucos trabalhos têm verificado esta possibilidade, mesmo em nível teórico.

O objetivo deste artigo é examinar os efeitos das políticas desacopladas previstas na Lei Agrícola Americana de 2002 (Farm Act 2002), especialmente em relação aos efeitos indiretos do risco nas decisões de produção. Especificamente, este estudo procurará analisar a existência de efeitos-renda e segurança nos instrumentos do Farm Act 2002, cujos impactos foram pouco estudados até o momento. Ao contrário de outros estudos, ênfase será dada na identificação do papel do índice de magnitude de auxílio nas decisões dos produtores.

O artigo é organizado da seguinte maneira: a seção dois analisa as políticas desacopladas contidas na lei agrícola norte-americana de 2002; a seção três descreve os efeitos sobre o risco e produção destas políticas; a seção quatro apresenta a metodologia e é seguida pela aplicação desta última no caso das políticas norte-americanas. A seção seis analisa os efeitos-renda e segurança e a seção sete apresenta as conclusões do artigo.

\section{2 - Políticas desacopladas na Política Agrícola norte-americana}

\section{A) Pagamentos diretos}

Os EUA adotaram os pagamentos diretos aos agricultores sob o nome de Production Flexibility Contracts (PFC) explicitados no Federal Improvement and Reform Act (FAIR Act) de 1996. Estes pagamentos, também conhecidos como Agriculture Market Transition Assistance (AMTA), consistiam em transferências anuais, por um período de seis anos, para os agricultores que possuíssem terras inscritas previamente em outros programas de apoio a agricultura (Base acreage) nas culturas de trigo, arroz, milho, cevada, aveia, sorgo e algodão. Estes pagamentos eram totalmente desacoplados da produção e dos preços correntes, dependendo apenas da área e produtividade histórica da cultura especificada 
no contrato. Além disso, havia total flexibilidade para plantar qualquer cultura na área contratada, independente daquela definida em contrato, ou seja, era possível receber AMTA para trigo e plantar milho na área contratada (WESTCOTT et alli, 2002). O montante transferido no período de 1996 a 2002 foi de US\$ 36 bilhões, o que representou cerca de 9\% da renda agrícola líquida no período.

Sob o Farm Act de 2002, os pagamentos diretos mantiveram as mesmas características dos PFC. A diferença fundamental é que sob o Farm Act de 2002, as taxas de pagamento (Payment rates) foram fixadas para toda a extensão do contrato, ao contrário dos PFC, que previam apenas os montantes estipulados para cada ano, posteriormente divididos por cultura.

Para o cálculo dos pagamentos diretos (Pd) sob o Farm Act de 2002, usa-se a seguinte fórmula (no caso do milho, por exemplo):

$\mathrm{Pd}_{\text {milho }}=(\text { Taxa de pagamento })_{\text {milho }} \mathrm{x}$ (Produtividade) $\left.)_{\text {milho }} \mathrm{x}[\text { (base acres) })_{\text {milho }} \mathrm{x} 0,85\right]$.

As taxas de pagamento são definidas pelo Congresso americano e pode-se notar que os pagamentos são feitos apenas para $85 \%$ da área contratada.

\section{B) Pagamentos anticíclicos}

Os pagamentos anticíclicos (CCP) foram criados pelo Farm Act de 2002 como forma de substituir os chamados Market Loss Assistance Payments (MLA). Os MLA foram criados em 1998 pelo Congresso americano como forma de compensar os agricultores pelos baixos preços das commodities observados naquele ano. Nos anos posteriores, vários pacotes emergenciais para diferentes produtos (leite, suínos, etc.) foram criados, totalizando cerca de US\$ 24 bilhões no período de 1999-2001. Para substituir estes programas ad hoc, instituíram-se os CCP. Este programa é uma espécie de re-edição do programa de preços meta que havia sido encerrado em 1996. Sua diferença fundamental está no fato de os pagamentos serem desacoplados da produção corrente: a base de cálculo são as áreas e produtividades históricas. Entretanto, os CCP não são desacoplados dos preços correntes: os pagamentos são feitos baseados na diferença entre o preço meta e o preço corrente (ou o preço 
mínimo, o que for maior). Assim, os pagamentos anticíclicos não podem ser incluídos na "caixa-verde" da Rodada Uruguai.

Os cálculos dos CCP são feitos da seguinte forma:

$\mathrm{CCP}_{\text {milho }}=(\text { Taxa de pagamento CCP })_{\text {milho }} \times(\text { Produtividade CCP })_{\text {milho }} \times[\text { (base acres })_{\text {milho }}$ $\mathrm{x} 0,85$ ]

(Taxa de pagamento CCP $)_{\text {milho }}=(\text { preço meta })_{\text {milho }}-(\text { Taxa do pagamento direto })_{\text {milho }}$ - (Preço atual ou preço mínimo, o que for maior) ${ }_{\text {milho }}$

\section{3 - Efeito das políticas desacopladas sobre o risco e a produção}

Os pagamentos diretos são conceitualmente diferentes dos tradicionais programas de suporte à agricultura usados anteriormente. Sua característica principal é ser desacoplado das decisões correntes de produção e dos preços correntes, ou seja, o montante recebido pelos agricultores não muda com variações no preço ou na quantidade produzida.

No caso dos programas de suporte de preço, ao contrário, os subsídios afetam diretamente as decisões de produção, pois modificam os preços recebidos pelos agricultores, influenciando assim os retornos marginais da produção. Dessa forma, retornos marginais mais altos estimulam o aumento da produção e acabam determinando muitas vezes quedas nos preços internacionais das commodities.

Entretanto, apesar de desacopladas, muitas políticas podem ter efeitos sobre a produção através de efeitos indiretos. Estes efeitos podem ser derivados de três causas principais:

a) Efeito-renda causando redução da aversão absoluta ao risco dos produtores: as transferências de renda podem causar uma diminuição da aversão ao risco nos produtores que apresentam aversão absoluta ao risco decrescente com a renda. Isto significa que, após a transferência de renda, os produtores estarão dispostos a assumir riscos maiores, aumentando a área plantada ou transferindo a produção para culturas mais arriscadas e com maior retorno.

b) Efeito-segurança: Este efeito é observado em políticas desacopladas da produção, mas não dos preços correntes. O programa governamental pode afetar o risco enfrentado pelos agricultores. Isso ocorre sempre que o montante varie com a fonte de incerteza do produtor (na maioria 
das vezes, o preço na colheita) como, por exemplo, no caso dos pagamentos anticíclicos. A lógica é que uma política que diminua o risco ou a variância da receita esperada terá efeitos positivos na produção dos produtores avessos ao risco.

c) Efeito-Investimento: Muitos agricultores apresentam restrições à tomada de crédito no que se refere ao montante obtido ou às taxas de juros oferecidas. Isto muitas vezes resulta num nível de investimento na propriedade abaixo do nível ótimo, significando que os retornos marginais dos investimentos adicionais seriam maiores que seus custos. As transferências de renda podem diminuir este problema, permitindo um maior investimento, seja via capital próprio, seja devido à maior disponibilidade de crédito (e também ao menor custo) advinda do oferecimento de melhores garantias ou de um fluxo mais estável da receita. Este maior investimento certamente tem efeitos na produção, pois permite a adoção de tecnologias mais produtivas disponíveis para os agricultores mais capitalizados.

Alguns trabalhos procuraram incorporar estes efeitos na análise das decisões dos produtores. Chavas \& Holt (1990) encontraram resultados, através de um modelo de resposta de oferta, indicando a importância do efeito-renda nas decisões de plantio de milho e soja nos EUA. Com base nesse resultado, Young \& Westcott (2000) estimaram que os pagamentos diretos (PFC) poderiam aumentar a área plantada nos EUA em cerca de 570 mil acres anualmente, um efeito pequeno relativo à área plantada total (247 milhões de acres). Adams et al. (2001) estimaram os efeitos dos pagamentos diretos (PFC) e do market loss assistance através de dados de painel e encontraram evidências fracas de efeitos positivos destas transferências de renda na área plantada total nos EUA.

Na próxima seção, os efeitos renda e segurança serão analisados através de um modelo que incorpore o papel do risco na decisão dos agricultores. O efeito investimento, que exige outro tipo de modelo, será objeto de pesquisa e publicação posterior.

\section{4 - Metodologia}

A metodologia utilizada neste trabalho foi desenvolvida por Hennessy (1998), que considerou, num referencial neoclássico de análise, a 
existência de um produtor maximizador de utilidade do lucro esperado. Suponha, assim, que o produtor maximize:

$$
\operatorname{Max}_{\alpha} \int_{a}^{b} U[\pi(\alpha, \varepsilon)] d H(\varepsilon)
$$

Em que:

$$
\begin{aligned}
& \pi(\alpha, \varepsilon)=\text { função lucro do produtor } \\
& \alpha=\text { variável de escolha do produtor } \\
& \varepsilon=\text { variável estocástica }(\varepsilon>0 \text { no intervalo [a,b]) } \\
& H(\varepsilon)=\text { função de distribuição cumulativa de } \varepsilon \\
& U(.) \text { é monotônica e crescente }
\end{aligned}
$$

A condição de primeira ordem (CPO) para maximização é dada por:

$$
\int_{a}^{b} U_{\pi}[\pi(\alpha, \varepsilon)] \pi_{\alpha}(\alpha, \varepsilon) d H(\varepsilon)=0
$$

A solução é dada para $\alpha=\alpha^{*}$. A condição de segunda ordem (CSO) é dada por:

$$
\Delta=\int_{a}^{b}\left\{U_{\pi \pi}[\pi(\alpha, \varepsilon)]\left[\pi_{\alpha}(\alpha, \varepsilon)\right]^{2}+U_{\pi}[\pi(\alpha, \varepsilon)] \pi_{\alpha \alpha}(\alpha, \varepsilon)\right\} d H(\varepsilon)<0
$$

Pressupõe-se que esta condição para maximização seja observada, isto é, que a expressão acima seja negativa. Define-se $m(\beta, \varepsilon)$ como uma função positiva representando a política de auxílio governamental aos produtores, em que $\beta$ representa um índice da magnitude do auxílio. No caso dos pagamentos diretos do governo americano, $\beta$ pode ser entendido como as taxas de pagamento (payment rates) fixadas pelo governo, de tal forma que $m_{\beta}(\beta, \varepsilon)>0$. Como $m(\beta, \varepsilon)>0$, a função de distribuição de lucros com o auxílio governamental desloca-se para a direita em relação à função de distribuição de lucros sem auxílio para os mosmos $\varepsilon$ avaliados para $\alpha^{*}$. Assim, tem-se:

$$
\pi^{+}(\alpha, \beta, \varepsilon)=\pi(\alpha, \varepsilon)+m(\beta, \varepsilon)
$$

Em que: 


$$
\begin{aligned}
& \pi^{+}(\alpha, \beta, \varepsilon)=\text { lucro aumentado pelo auxílio governamental } \\
& \pi(\alpha, \varepsilon)=\text { lucro de mercado }
\end{aligned}
$$

A forma aditiva observada acima só é possível para políticas desacopladas da produção, de forma que os lucros de mercado não dependam da variável $\beta$. Transformando (2), tem-se:

$$
\int_{a}^{b} U_{\pi}\left[\pi^{+}(\alpha, \beta, \varepsilon)\right] \pi_{\alpha}(\alpha, \varepsilon) d H(\varepsilon)=0
$$

O que se quer demonstrar é o efeito de um aumento das políticas governamentais sobre as decisões de produção dos agricultores, ou seja, é necessário determinar o sinal de $\frac{\partial \alpha}{\partial \beta}$. Assim, diferenciando parcialmente (3) com relação a $\alpha$ e $\beta$ e re-arranjando os termos, tem-se:

$$
\frac{\partial \alpha}{\partial \beta}=-\frac{1}{\Delta} \int_{a}^{b} U_{\pi \pi}\left(\pi^{+}\right) \pi_{\alpha} m_{\beta} d H(\varepsilon)
$$

Sabe-se que o coeficiente de aversão absoluta ao risco é dado por:

$$
R\left(\pi^{+}\right)=-\frac{U_{\pi \pi}\left(\pi^{+}\right)}{U_{\pi}(\pi)}
$$

Substituindo (5) em (4):

$$
\frac{\partial \alpha}{\partial \beta}=\frac{1}{\Delta} \int_{a}^{b} R\left(\pi^{+}\right) m_{\beta} U_{\pi}\left(\pi^{+}\right) \pi_{\alpha} d H(\varepsilon)
$$

Fazendo $R\left(\pi^{+}\right) m_{\beta}=J(\varepsilon)$ e integrando (6) por partes:

$$
\frac{\partial \alpha}{\partial \beta}=\frac{1}{\Delta}\left[\left.J(\varepsilon) \int_{a}^{v} U_{\pi}\left(\pi^{+}\right) \pi_{\alpha} d H(\varepsilon)\right|_{v=a} ^{v=b}-\iint_{a} U_{\pi}\left[\pi^{+}\right] \pi_{\alpha} d H(\varepsilon) \frac{d J(v)}{d v} d v\right]
$$

Em que $v$ é a representação de $\varepsilon$ no processo de integração. Pela equação (3), o primeiro termo do lado direito de (7) iguala-se a zero. Assim, a análise do impacto das políticas de auxílio desacopladas sobre a produção depende da determinação do sinal da integral dupla. 


\section{5 - Determinação do sinal de $\frac{\partial \alpha}{\partial \beta}$ para as políticas desacopladas da lei agrícola americana $\partial \beta$}

\section{a) Pagamentos diretos}

No caso dos pagamentos diretos, a variável de escolha $\alpha$ pode ser entendida como a extensão da área a ser plantada. A variável estocástica $\varepsilon$ é o preço de mercado na época da colheita e a variável $\beta$ pode ser entendida como a taxa de pagamento (payment rate) estabelecida pelo governo para cada cultura. Para analisar o sinal de (7), pode-se proceder à análise da cada termo separadamente:

- $\frac{d J(v)}{d v}-J(\varepsilon)$ foi definido como $R\left(\pi^{+}\right) m_{\beta}$. Ao se considerar que os

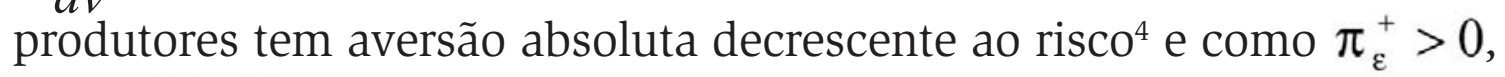
então $R\left(\pi^{+}\right)$é uma função positiva mas decrescente em $\varepsilon$. Para $m_{\beta}$, que é o impacto marginal das taxas de pagamento sobre o montante de auxílio, sabe-se que, no caso dos pagamentos diretos, ela é invariante a mudanças nos preços correntes, ou seja, $m_{\beta \varepsilon}=0$. Assim, $m_{\beta}$ é constante em relação a $\varepsilon$ e o sinal de $\underline{d J(v)}$ depende apenas do sinal de $\frac{\partial R\left(\pi^{+}\right)}{\partial \varepsilon}<0$. Assim, $\frac{d J(v)}{d v}<0$. $d v$

- $\int_{a}^{v} U_{\pi}\left[\pi^{+}\right] \pi_{\alpha}\left(\alpha^{*}, \varepsilon\right) d H(\varepsilon)$ - A análise deste termo envolve a definição do sinal de $\pi_{\alpha \varepsilon}(\alpha, \varepsilon)$. Este termo capta o impacto dos preços de mercado na época da colheita no impacto marginal da área plantada nos lucros. Este termo é claramente positivo, uma vez que um aumento nos preços aumenta o retorno marginal por hectare plantado. Assim, $\pi_{\alpha \varepsilon}(\alpha, \varepsilon)>0$. Dessa forma, para $\varepsilon=a, U_{\pi}\left[\pi^{+}\right] \pi_{\alpha}\left(\alpha^{*}, \varepsilon\right)$ é negativo e para $\varepsilon=b, U_{\pi}\left[\pi^{+}\right] \pi_{\alpha}\left(\alpha^{*}, \varepsilon\right)$ é positivo e há um valor máximo de $\varepsilon$ para o qual $\pi_{\alpha}\left(\alpha^{*}, \varepsilon\right)=0$. Disso decorre que $\int_{a}^{v} U_{\pi}\left[\pi^{+}\right\rceil \pi_{*}\left(\alpha^{*}, \varepsilon\right) d H(\varepsilon)$ é negativo e decrescente em $v$ para o intervalo $\left[\alpha, \varepsilon^{*}\right)$ e negativo e crescente em $v$ para o intervalo $\left[\varepsilon^{*}, b\right]$ pois, de acordo com a equação (3), o valor máximo é zero e é atingido quando $v=b$. Assim, $\int_{a}^{v} U_{\pi}\left[\pi^{+}\right] \pi_{\alpha}\left(\alpha^{*}, \varepsilon\right) d H(\varepsilon)<0$.

${ }^{4}$ Chavas e Holt (1990) encontraram evidências de aversão absoluta ao risco decrescente em relação à renda para os produtores de soja e milho nos EUA. 
Dessa forma, o sinal de (7) é composto pela integral do produto de dois termos negativos, ou seja, por um valor positivo, multiplicado por $-\frac{1}{\Delta}$. Como $\Delta<0$, pode-se concluir que, para a caso dos pagamentos diretos, $\frac{\partial \alpha}{\partial \beta}>0$, isto é, um aumento das transferências governamentais via pagamentos diretos tem um impacto positivo sobre a extensão da área plantada e, conseqüentemente, sobre a produção.

\section{b) Pagamentos anticíclicos}

No caso dos pagamentos anticíclicos, a variável de escolha $\alpha$ pode ser entendida novamente como a extensão da área a ser plantada e a variável estocástica $\varepsilon$, como o preço de mercado na época da colheita. Já a variável $\beta$ pode ser entendida como o preço meta estabelecido pelo governo para cada cultura. Analisando novamente os sinais:

- $\frac{d J(v)}{d v}$ - Considerando novamente que os produtores tem aversão absoluta decrescente ao risco, a diferença na análise em relação aos pagamentos diretos se dá no sinal de $m_{\beta \varepsilon}$. Isso ocorre porque, no caso dos CCP, o impacto marginal do preço-meta sobre o montante de auxílio não é invariante a mudanças nos preço correntes, isto é, quanto menor o preço corrente, maior o impacto marginal dos preços meta sobre a função lucro. Assim, $m_{\beta \varepsilon}<0$, o que reforça o sinal negativo de $\frac{\partial R\left(\pi^{+}\right)}{\partial \varepsilon}$ e faz com que $\frac{d J(v)}{d v}$ seja negativo e em valor absoluto maior que no caso dos pagamentos diretos.

- $\int_{a}^{v} U_{\pi}\left[\pi^{+}\right] \pi_{\alpha}\left(\alpha^{*}, \varepsilon\right) d H(\varepsilon)$ - A análise do sinal é idêntico ao caso dos pagamentos diretos, pois para os CCP também se observa $\pi_{\alpha \varepsilon}(\alpha, \varepsilon)>0$, ou seja, um aumento nos preços de mercado aumenta o retorno marginal por hectare plantado. Assim, $\int_{a}^{\nu} U_{\pi}\left[\pi^{+}\right] \pi_{\alpha}\left(\alpha^{*}, \varepsilon\right) d H(\varepsilon)<0$.

Assim, os efeitos sobre a área plantada e a produção de um aumento nos preços-meta também é positivo, isto é, $\frac{\partial \alpha}{\partial \beta}>0$, mesmo que os pagamentos anticíclicos sejam desacoplados da produção atual, baseando-se 
em áreas e produtividades históricas. Além disso, a magnitude de $\frac{\partial \alpha}{\partial \beta}$ é maior no caso dos CCP do que no caso dos pagamentos diretos. $\partial \beta$

\section{6 - Efeito-Renda e Efeito-Segurança}

Hennessy (1998) divide os efeitos sobre a produção em dois tipos: o efeito-renda e o efeito segurança (insurance effect). $\mathrm{O}$ efeito renda decorre da aversão absoluta decrescente ao risco e do fato de que $\frac{\partial R\left(\pi^{+}\right)}{\partial \varepsilon}<0$, pois quanto maior $\varepsilon$, maior o lucro $\pi^{+}$. O que ocorre é que um aumento de renda induzido pelo auxílio governamental muda a distribuição da função lucro para uma região de menor aversão ao risco da função de utilidade dos produtores. Isso causa, por sua vez, um aumento da variável de escolha dos produtores (no exemplo, extensão de área plantada) e, conseqüentemente, do total produzido.

Já o efeito-segurança aparece pela redução da variabilidade da receita dos produtores causada pelo auxílio governamental. Isto ocorre quando $m_{\beta \varepsilon}<0$, ou seja, quando uma redução no preço de mercado é compensada pelo aumento do montante transferido pelo governo.

No caso do programa de pagamentos diretos do governo americano, apenas o efeito renda está presente, enquanto o programa de pagamentos anticíclicos apresenta efeitos renda e segurança. Isso ocorre porque os pagamentos diretos são desacoplados dos preços correntes, ou seja, o montante transferido não muda com variações dos $\operatorname{preços}\left(m_{\beta \varepsilon}=0\right)$. Já no caso dos pagamentos anticíclicos, uma diminuição nos preços correntes é compensada por um aumento das transferências governamentais $\left(m_{\beta \varepsilon}<0\right)$, diminuindo a variabilidade da receita e reforçando os efeitos sobre a produção do efeito renda também presente.

Entretanto, os resultados encontrados acima dependem da hipótese de que os agricultores anresentem aversão absoluta decrescente ao risco, ou seja, de que $\frac{\partial R\left(\pi^{+}\right)}{\partial \pi^{+}}<0$. Caso a função utilidade apresente comportamentn diferente, como por exemplo, aversão absoluta ao risco constante $\left(\frac{\partial R\left(\pi^{+}\right)}{\partial \pi^{+}}=0\right)$, o efeito renda desaparece. Assim, sob este último exemplo, não existem mais efeitos dos pagamentos diretos sobre a 
produção , isto é, $\frac{\partial \alpha}{\partial \beta}=0 \cdot{ }^{5}$ No caso dos pagamentos anticíclicos, mesmo com aversãn ahcnluta ao risco constante, ainda existem efeitos sobre a produção $\left(\frac{\partial \alpha}{\partial \beta}>0\right)$, pois resta o efeito segurança, já que $m_{\beta \varepsilon}<0 .{ }^{6}$

\section{7 - Conclusão}

Nos últimos anos, esforços têm sido feitos no sentido de modificar as políticas de apoio à agricultura de forma a minimizar as políticas distorcivas sobre a produção e o comércio internacional. Ênfase tem sido dada às políticas de transferências diretas de renda aos agricultores, consideradas neutras em relação às decisões de produção corrente. Esta pressuposição está implícita até nas resoluções da Rodada Uruguai do GATT, que considerou este tipo de política de apoio isenta de qualquer compromisso de redução, incluindo-a no que se denominou "caixa-verde".

Este estudo mostra que, ao contrário, as decisões de produção correntes são afetadas pelas políticas de transferência de renda, especialmente através dos efeitos diretos sobre a percepção de risco dos agricultores. Estes efeitos, divididos em efeito-renda e efeito-segurança, estimulam o aumento da área plantada e assim, têm efeitos sobre a produção e os preços das commodities. No caso dos pagamentos diretos, apenas o efeito renda está presente. Já nos anticíclicos, mesmo desacoplados da produção corrente, apresentam, além do efeito renda, também o efeito-segurança. Outros efeitos não examinados neste trabalho, como aquele sobre o nível de investimentos na propriedade após a transferência, contribuem para aumentar os impactos dos pagamentos sobre a produção.

As implicações destes resultados são muito importantes. Nos últimos dez anos, os países desenvolvidos têm modificado de forma gradual sua política agrícola, transferindo recursos dos programas de suporte de pre-

${ }^{5}$ É fácil ver que, neste caso, $\frac{d J(v)}{d v}=0$, pois $m_{\beta \varepsilon}=0$ e $\frac{\partial R\left(\pi^{+}\right)}{\partial \varepsilon}=0$, o que faz com que a expressão (7) seja nula.

${ }^{6}$ Neste caso, $\frac{d J(v)}{d v}<0$, pois $m_{\beta \varepsilon}<0$, fazendo com que $\frac{\partial \alpha}{\partial \beta}>0$. 
ços para as transferências diretas. Esta mudança tem sido defendida na medida que, aparentemente, reduz o impacto distorcivo sobre produção, preços e comércio internacional, ao mesmo tempo em que permite a continuação de políticas de garantia de renda para o setor rural. Entretanto, considerando o papel da redução do risco na tomada de decisão dos agricultores, o papel distorcivo das transferências diretas não pode ser descartado. É necessário, assim, avaliar até que ponto a magnitude desses efeitos se compara àqueles derivados das políticas de suporte de preços e se é possível aperfeiçoar o mecanismo de transferência de forma a minimizar os efeitos sobre a produção.

De qualquer forma, a suposta neutralidade das políticas desacopladas da produção e dos preços pode ser descartada. Na medida em que os países desenvolvidos continuarem subsidiando seus agricultores, seja via suporte de preços, seja via transferências diretas, os impactos sobre os preços e o comércio internacional continuarão a existir.

\section{8 - Referências bibliográficas}

ADAMS, G.; WESTHOFF, P.; WILLOTT, B.; YOUNG II, R.E. “ Do “Decoupled" Payments affect U.S. Crop Area? Preliminary Evidence from 1997-2000" American Journal of Agricultural Economics, Vol. 83, No. 5, 2001, pp. 1190-1195.

BURFISHER M.E. \& HOPKINS J. (ed.) Decoupled Payments: Household Income Transfers in Contemporary U.S. Agriculture. Market and Trade Economics Division, Economic Research Service, U.S. Department of Agriculture. Agricultural Economic Report No. 822, 2003.

CHAVAS, J.P. \& HOLT M.T. "Acreage Decisions Under Risk: The Case of Corn and Soybeans.” American Journal of Agricultural Economics, Volume 72, August 1990, pp. 529- 538.

HENNESSY, D.A. “The Production Effects of Agricultural Income Support Policies under Uncertainty," American Journal of Agricultural Economics, Vol. 80, No. 1,1998, pp. 46-57.

Organization for Economic Cooperation and Development.(OECD) Decoupling: A Conceptual Overview, OECD papers No. 10, OECD, Paris, 2001. 
TEIXEIRA, E.C. Política de garantia de renda. In: SANTOS, M.L. e VIEIRA, W.C. (Editores) A Agricultura na virada do milênio: Velhos e novos desafios.Viçosa, 2000.

YOUNG, C.E. \& WESTCOTT, P.C. "How Decoupled is U.S. Agricultural Support for major crops" American Journal of Agricultural Economics, Vol. 82, No. 3, 2000, pp. 762-767.

WESTCOTT, P.C., YOUNG, C.E., PRICE, J. M. The 2002 Farm Act: Provisions and Implications for Commodity Markets/AIB-778 Economic Research Service/USDA, 2002.

Recebido em setembro de 2004 e revisto em janeiro de 2005. 